ARTICLE

https://doi.org/10.1038/s41467-019-10981-9

\title{
Reactivity of shape-controlled crystals and metadynamics simulations locate the weak spots of alumina in water
}

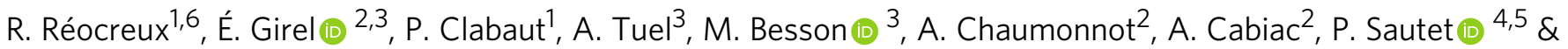 \\ C. Michel (iD ${ }^{1}$
}

The kinetic stability of any material in water relies on the presence of surface weak spots responsible for chemical weathering by hydrolysis. Being able to identify the atomistic nature of these sites and the first steps of transformation is therefore critical to master the decomposition processes. This is the challenge that we tackle here: combining experimental and modeling studies we investigate the stability of alumina in water. Exploring the reactivity of shape-controlled crystals, we identify experimentally a specific facet as the location of the weak spots. Using biased ab initio molecular dynamics, we recognize this weak spot as a surface exposed tetra-coordinated $\mathrm{Al}$ atom and further provide a detailed mechanism of the first steps of hydrolysis. This understanding is of great importance to heterogeneous catalysis where alumina is a major support. Furthermore, it paves the way to atomistic understanding of interfacial reactions, at the crossroad of a variety of fields of research.

\footnotetext{
${ }^{1}$ Univ Lyon, Ens de Lyon, CNRS UMR 5182, Université Claude Bernard Lyon 1, Laboratoire de Chimie, 69342 Lyon, France. ${ }^{2}$ Direction Catalyse et Séparation, IFP Energies nouvelles, Rond-point de l'échangeur de Solaize, BP 3, 69360 Solaize, France. ${ }^{3}$ Institut de Recherches sur la Catalyse et I'Environnement de Lyon, IRCELYON, CNRS UMR 5256-Univ. Lyon 1, 2, avenue Albert Einstein, 69626 Villeurbanne, France. ${ }^{4}$ Department of Chemical and Biomolecular Engineering, University of California Los Angeles, Los Angeles, CA 90095, USA. ${ }^{5}$ Department of Chemistry and Biochemistry, University of California Los Angeles, Los Angeles, CA 90095, USA. 'Present address: Thomas Young Centre and Department of Chemical Engineering, University College London, Roberts Building, Torrington Place, London WC1E 7JE, UK. Correspondence and requests for materials should be addressed to P.S. (email: sautet@ucla.edu) or to C.M. (email: carine.michel@ens-lyon.fr)
} 
T he kinetic stability of solids in water is governed by their reactivity at the interface. Being able to understand and control their surface stability and desired properties is therefore at the heart of a variety of research fields: kinetics of drug release ${ }^{1}$, corrosion of metals and alloys ${ }^{2}$, lithium batteries ${ }^{3}$, geochemistry ${ }^{4}$ with in particular the re-equilibration of solid phases in presence of a liquid ${ }^{5}$, water treatment ${ }^{6}$, heterogeneous catalysis $^{7,8}$, from the preparation ${ }^{9,10}$, the utilization to the degradation of the catalyst ${ }^{11,12}$, etc. Several parameters have been identified as key in all these fields: the solid phase (which polymorph $)^{13}$, the nature of the surface exposed (kink, rugosity $)^{4}$, and the species in solutions (additives, ions, $\mathrm{pH})^{14-16}$.

For instance, alumina is an oxide that is used in catalysis as a support for metallic nanoparticles ${ }^{17}$. Its $\gamma$-allotrope $\left(\gamma-\mathrm{Al}_{2} \mathrm{O}_{3}\right)$ in particular has remarkable properties as a support, long proven in the development of gas phase transformations ${ }^{18-21}$. However, water has detrimental effects on $\gamma-\mathrm{Al}_{2} \mathrm{O}_{3}$, either as a liquid ${ }^{12}$ or even as steam ${ }^{22}$. While its polymorph $\alpha-\mathrm{Al}_{2} \mathrm{O}_{3}$ is stable, $\gamma-\mathrm{Al}_{2} \mathrm{O}_{3}$ indeed transforms into various sorts of bulk hydroxides $\left(\mathrm{AlO}_{\mathrm{x}} \mathrm{H}_{\mathrm{y}}\right)$ thereby seriously damaging the catalyst (decrease of surface area, sintering and encapsulation of catalytic particles $)^{23-25}$. Albeit not fully described at the atomic scale yet, the decomposition of $\gamma$ $\mathrm{Al}_{2} \mathrm{O}_{3}$ was shown to proceed, at the macroscopic scale, through a sequential mechanism involving first dissolution of $\mathrm{Al}$ atoms, and then precipitation thereof into the undesired hydroxides ${ }^{16,24}$. These structural changes can be retarded by tuning the surface chemical composition: silica deposition ${ }^{26}$, presence of metallic nanoparticles ${ }^{12}$, or impregnation with metal ions ${ }^{27}$. They are also impacted by the content of the solution: $\mathrm{pH}^{24}$ but also presence of polyols ${ }^{28}$ or polyphenols ${ }^{25}$. The mechanism of action of these additives is believed to lie in their chemisorption on the surface that would make $\gamma-\mathrm{Al}_{2} \mathrm{O}_{3}$ water-resistant ${ }^{28,29}$. However little is known on which exposed facet(s) and what site(s) need to be particularly targeted for protection. The optimization of the structure of inhibitors is therefore a challenging task since the exact mechanism of the decomposition of $\gamma-\mathrm{Al}_{2} \mathrm{O}_{3}$ in liquid water remains unknown. Whilst the sub-nanometric description of $\gamma$ $\mathrm{Al}_{2} \mathrm{O}_{3}$ is difficult to reach experimentally, molecular simulations have allowed for the development of insightful atomistic models for amorphous ${ }^{30}$ and nano-crystalline ${ }^{31} \gamma-\mathrm{Al}_{2} \mathrm{O}_{3}$. The model proposed by Digne et al. ${ }^{31}$, which was obtained from density functional theory (DFT) calculations, has proven to depict properly the reactivity of the surface under various realistic gas phase conditions ${ }^{19}$. It is only recently that Ngouana-Wakou et al..$^{32}$ and Réocreux et al..$^{33}$ have explored the interaction of $\gamma$ $\mathrm{Al}_{2} \mathrm{O}_{3}$ with liquid water, performing ab initio molecular dynamics (AIMD) simulations. Although these simulations give insights on the relative affinity of water for the different facets and the structuration and dynamics of the liquid at the interface, they cannot capture the details of the decomposition mechanism. Al-O bond formation and scissions are indeed rare events on the time scale achievable today with AIMD. To overcome this limitation and force the system to react along a chosen reaction coordinate, biased AIMD simulations are required. Ab initio metadynamics is an example of such methods ${ }^{34}$, and has been commonly used to model the reactivity between molecules in homogeneous media $^{35,36}$ or between isolated molecules and a solid surface ${ }^{37,38}$. Although common using classical force field for crystal growth and dissolution of molecular and ionic crystals ${ }^{39,40}$, metadynamics has never been used to describe a reactive interface involving bonds with a partial covalent character (typically here $\mathrm{Al}-\mathrm{O}$ and $\mathrm{O}-\mathrm{H}$ bonds) hence requiring an ab initio description.

Here, we combine experiments and theory to provide the atomistic mechanism for the early-stage decomposition of $\gamma$ $\mathrm{Al}_{2} \mathrm{O}_{3}$ in liquid water. We experimentally demonstrate that the decomposition is initiated at the (110) facet by exposing four samples of various shapes of nanoparticles to a hydrothermal treatment in the presence of aqueous solution of inhibitors (xylitol and sorbitol) with varying concentrations. Performing ab initio metadynamics simulations, we probe the reactivity of the (110)/water interface and identify specific aluminum tetrahedral centers that are particularly reactive with water. We show that interfacial water molecules are involved in the mechanism, both as reactants for the hydration of aluminum and as intermediates for the proton reshuffling required by the decomposition mechanism. We show that the substitution of chemisorbed water molecules on the surface with xylitol locally renders the surface more hydrophobic and pushes water molecules away from the water-sensitive $\mathrm{Al}$ sites.

\section{Results}

Identification of the facet whence the decomposition initiates. $\gamma-\mathrm{Al}_{2} \mathrm{O}_{3}$ particles mainly expose three facets commonly referred to with their set of Miller indices: the predominant and hydrophilic (110) facet, the less hydrophilic (100) facet, and the (111) facet (see Fig. 1a). In order to identify the facet(s) involved in the decomposition of $\gamma-\mathrm{Al}_{2} \mathrm{O}_{3}$, we have prepared four samples containing particles of different shapes (see Fig. 1g-j). These four samples therefore show four distinct proportions of facet areas, which have been quantified using X-ray diffraction (XRD) (see Supplementary Note 1). We have had them undergo hydrothermal treatment (i.e., $200^{\circ} \mathrm{C}, 2 \mathrm{~h}, 14 \mathrm{bar}$ autogeneous pressure) using various aqueous solutions of sorbitol (represented in Fig. 1b), a known inhibitor, and xylitol (represented in Fig. 1c), a shorter sugar polyol. We have observed a very strong alumina stabilization in the presence of both sorbitol or xylitol, suggesting that the minimal $-(\mathrm{CHOH})_{5}-$ sequence, common to those two polyols, is essential to protect alumina from water. This is consistent with the work by Ravenelle et al. ${ }^{28}$ showing that sorbitol is a good inhibitor while glycerol (C3polyol) is not as efficient. To go beyond this qualitative statement, we have determined for each sample the minimum surface coverage of polyol at which the decomposition of alumina gets inhibited, that is no hydroxides can be detected by XRD after hydrothermal treatment (see Supplementary Note 2). This quantity, referred to as inhibiting coverage, is reached for concentrations of $4 \mathrm{~g} \mathrm{~L}^{-1}$ of polyols for $2 \mathrm{~g}$ of alumina in $50 \mathrm{~mL}$ of solution and varies from 0.15 to $0.30 \mathrm{~nm}^{-2}$ for xylitol, and from 0.20 to $0.35 \mathrm{~nm}^{-2}$ for sorbitol, depending on the shape of the nanoparticles (see Supplementary Table 4). This strong variation of the inhibiting coverage with the distribution of facet surface areas (up to a factor of two in the case of xylitol) indicates that each facet does not interact equally with the two polyols.

In the case of a specific adsorption on one facet only, the sodetermined inhibiting coverage increases linearly with the fractional surface area of this facet with a zero-intercept (see Supplementary Note 4). As shown in Fig. 1d-f, this is only with the fractional surface area of the hydrophilic (110) facet that the inhibiting coverage of both sorbitol and xylitol correlates with a zero-intercept function. We cannot establish such correlations with the other fractional surface areas. Following the same kind of reasoning, we show in Supplementary Note 5 that edges and kinks are unlikely to be involved as major active sites for hydrolysis. Therefore, the adsorption of sorbitol or xylitol is specific to the (110) facet and allows for the total inhibition of the decomposition of $\gamma-\mathrm{Al}_{2} \mathrm{O}_{3}$ in liquid water at similar partial coverage of 0.36 and $0.44 \mathrm{~nm}^{-2}$ of (110) facet for xylitol and sorbitol, respectively (slopes on Fig. 1d). As a corollary, we can infer that the (110) facet must exhibit sites responsible for the hydrolysis of $\gamma-\mathrm{Al}_{2} \mathrm{O}_{3}$ and that the reactivity of these sites with water can be limited or even suppressed upon the adsorption of sorbitol or xylitol. 


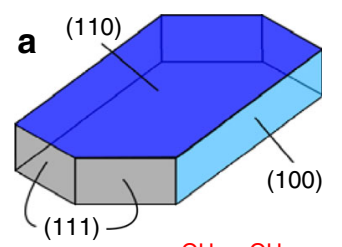

b

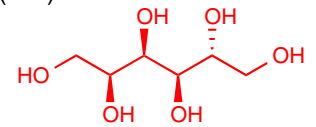

C<smiles>OC[C@@H](O)C(O)[C@H](O)CO</smiles>

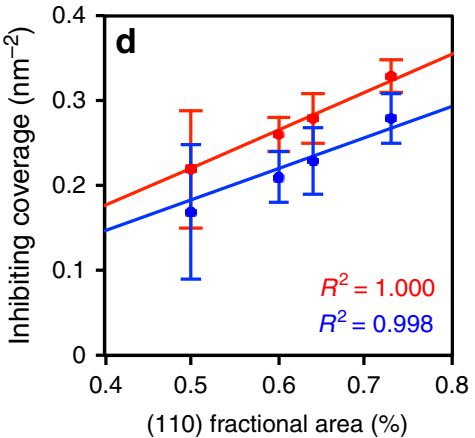

(110) fractional area (\%)

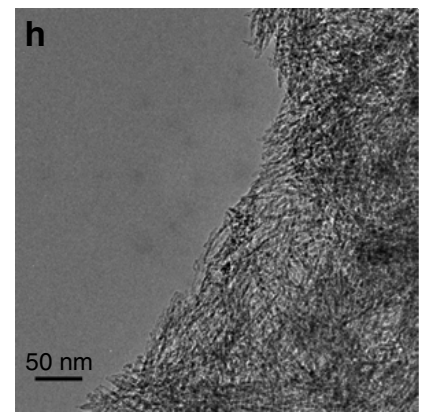

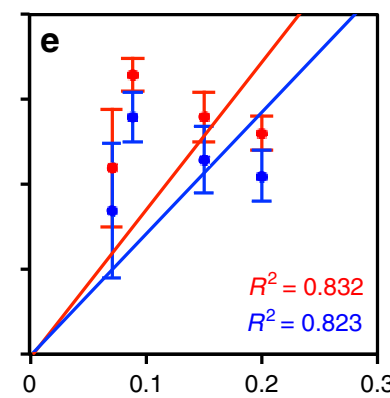

(100) fractional area (\%)

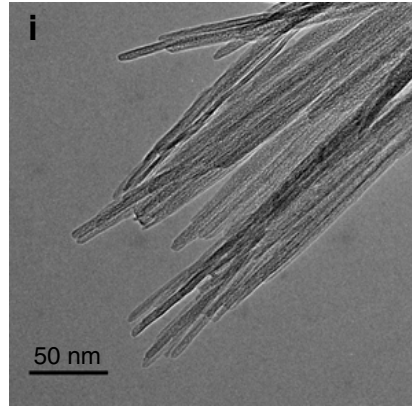

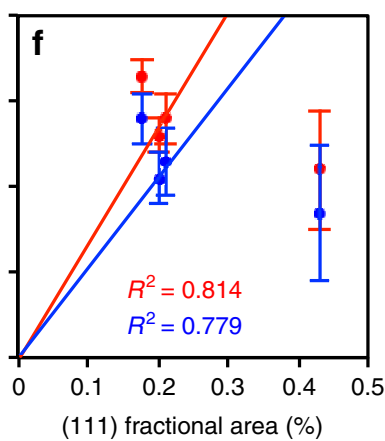

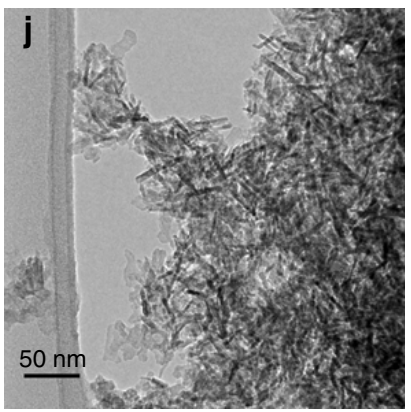

Fig. 1 Implication of the (110) facet in the decomposition of $\gamma-\mathrm{Al}_{2} \mathrm{O}_{3}$. a General topology of a $\gamma$ - $\mathrm{Al}_{2} \mathrm{O}_{3}$ nanoparticle exhibiting three major facets: (110) in dark blue, (100) in light blue and (111) in gray. b, c Structures of two inhibitors: b, sorbitol (red) and c, xylitol (blue). d, e, f Comparisons between the inhibiting coverage (sorbitol in red, xylitol in blue) and the fraction of exposed (110) (d), (111) (e), and (100) (f) surface areas. The experimental data sets are fitted to the zero-intercept linear model derived in Supplementary Note 4. The fitted curves are represented as straight lines. A correlation

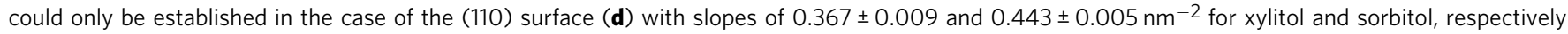
$\left(R^{2}>0.99\right)$. Error bars are calculated from high-performance liquid chromatography (HPLC) analysis standard deviation (see Supplementary Note 3 ).

$\mathbf{g}, \mathbf{h}, \mathbf{i}, \mathbf{j}$ Transmission electronic microscopy images of the four differently shaped $\gamma-\mathrm{Al}_{2} \mathrm{O}_{3}$ nanoparticles ordered in increasing (110) fractional area: plates $(\mathbf{g})$, fibers $(\mathbf{h})$, rods $(\mathbf{i})$, and commercial $\gamma-\mathrm{Al}_{2} \mathrm{O}_{3}(\mathbf{j})$

Identification of the atomic sites on the (110) facet whence the decomposition initiates. To gain atomistic insight and identify the sites involved in the decomposition of $\gamma-\mathrm{Al}_{2} \mathrm{O}_{3}$ on the (110) facet, we have performed ab initio simulations using our recently developed model for $\gamma-\mathrm{Al}_{2} \mathrm{O}_{3} /$ water interfaces ${ }^{33}$. The primitive cell of the model of the $\gamma-\mathrm{Al}_{2} \mathrm{O}_{3}(110)$ surface shows four different surface $\mathrm{Al}$ sites: two octahedral and two tetrahedral sites, referred to as $\mathrm{Al}_{(1)}$ and $\mathrm{Al}_{(2)}$ for the former and $\mathrm{Al}_{\alpha}$ and $\mathrm{Al}_{\beta}$ for the latter (see Fig. 2a, b). This surface is fully hydrated with five chemisorbed water fragments, the oxygen atoms of which are represented in blue in Fig. 2a, b. Some of these water molecules are dissociated, generating hydroxyl surface groups. Repeating the surface unit cell in the $\mathrm{x}$ and $\mathrm{y}$ directions, the resulting $\mathrm{p}(2 \times 2)$ slab is surmounted by a $20 \AA$ thick layer of liquid water that is not represented here for clarity. The iso-electric point of alumina being close to 8 , we consider pure neutral water. ${ }^{41,42}$ Since the decomposition of $\gamma-\mathrm{Al}_{2} \mathrm{O}_{3}$ transforms tetrahedral $\mathrm{Al}$ sites into octahedral $\mathrm{Al}$ centers, we have focused on the tetrahedral sites, $\mathrm{Al}_{\alpha}$ and $\mathrm{Al}_{\beta}$, and their reactivity with water (see Fig. $2 \mathrm{a}$ ). Both of them have a coordination number to alumina oxygen atoms $\left(\mathrm{CN}_{\mathrm{a}}\right)$ of three and a coordination number to water oxygen atoms $\left(\mathrm{CN}_{\mathrm{w}}\right)$ of one. Performing ab initio metadynamics, we have made each tetrahedral $\mathrm{Al}$ center specifically react along these two variables (see details in Methods). The statistical analysis of the variations of $\mathrm{CN}_{\mathrm{w}}$ and $\mathrm{CN}_{\mathrm{a}}$ allows for the construction of free energy landscapes (like the one given in Fig. 2c), the local depth of which assesses for the stability of the intermediates, transition states and products encountered along the course of the simulation (see details in Supplementary Note 6). Obtaining this mechanistic information is a key achievement in the quest of a better understanding of reactive interfaces and kinetic stability of solids in water. In the case of $\mathrm{Al}_{\alpha}$, the free energy landscape mainly shows one deep minimum at $\left(\mathrm{CN}_{\mathrm{a}}, \mathrm{CN}_{\mathrm{w}}\right)=(3,1)$, that is the initial structure (see Supplementary Fig. 6). Even within a span of $200 \mathrm{~kJ} \mathrm{~mol}^{-1}$, no transition state to escape that deep well towards other minima has been identified. This suggests that $\mathrm{Al}_{\alpha}$ is very unlikely to react with liquid water. Conversely, the free energy landscape for $\mathrm{Al}_{\beta}$ shows, over a span of $180 \mathrm{~kJ} \mathrm{~mol}^{-1}$, a variety of minima spread over the nodes of a well-defined checkered pattern and associated with a decreased number of bonds to alumina surface oxygen atoms and increased number with water oxygens (see Fig. $2 \mathrm{c}$ ). $\mathrm{Al}_{\beta}$ therefore progressively detaches from the oxygen network of $\gamma-\mathrm{Al}_{2} \mathrm{O}_{3}$ and is hydrated with the surrounding water molecules. A more detailed look at the free energy surface indicates that this hydration is sequential and follows an addition/elimination mechanism. It is only when $\mathrm{Al}_{\beta}$ has reached a total coordination number of 5 that an $\mathrm{Al}_{\beta}-\mathrm{O}-\mathrm{Al}$ bond cleavage occurs. Noticeably, five-coordinated species appear to be key intermediates in the decomposition of alumina as in the water-induced de-alumination of zeolites ${ }^{43}$. This holds true until $\left(\mathrm{CN}_{\mathrm{a}}, \mathrm{CN}_{\mathrm{w}}\right)=(1,4)$ where $\mathrm{Al}_{\beta}$ readily guests a water molecule to achieve an octahedral structure with $\left(\mathrm{CN}_{\mathrm{a}}\right.$, $\left.\mathrm{CN}_{\mathrm{w}}\right)=(1,5)$. This last structure obtained from the simulation is represented in Fig. 2e and shows how $\mathrm{Al}_{\beta}$ has been extracted from its initial position. Among the water molecules in the first coordination sphere, three of them were initially present on the surface as chemisorbed water molecules and the two others were physisorbed water molecules. Proton transfers are not included in the general reaction coordinates we designed. Nevertheless, the inspection of the trajectory shows that these early-stage steps of 
a

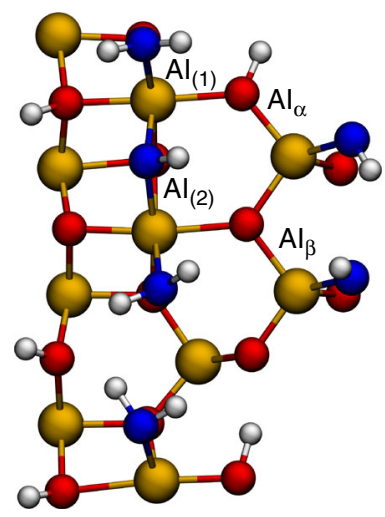

b

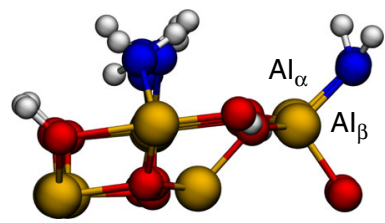

C

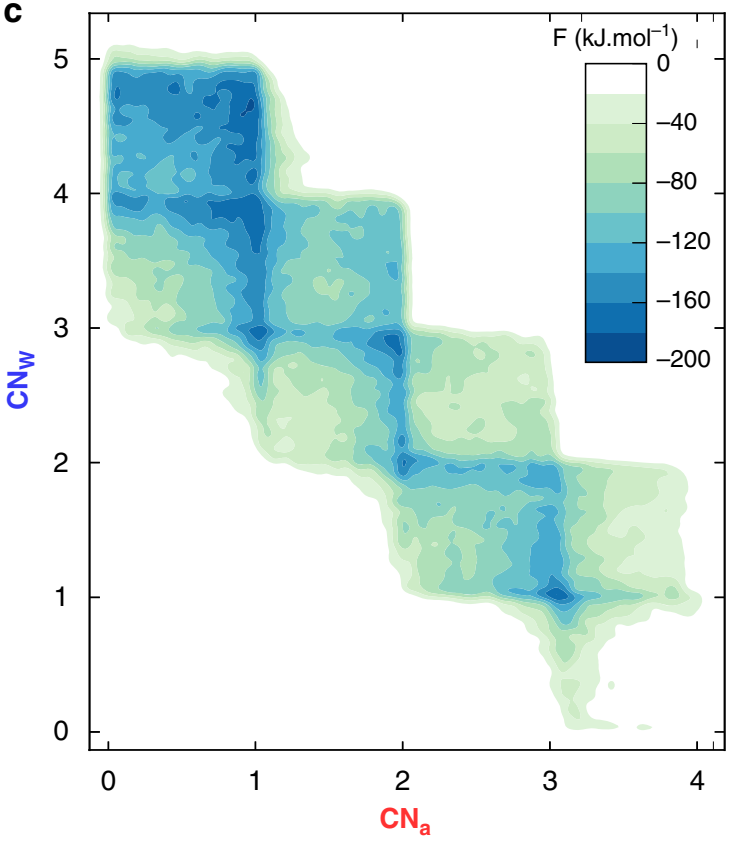

d

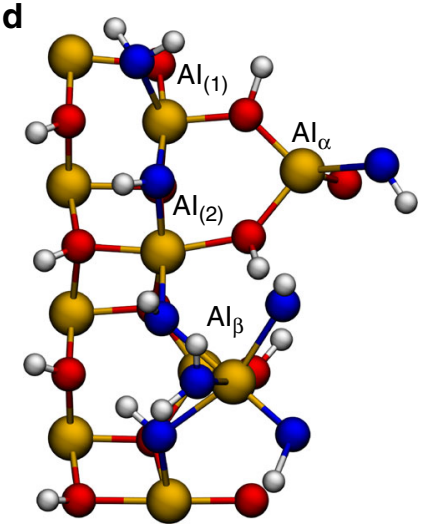

e

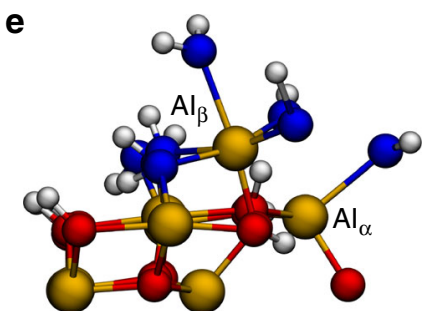

f $\mathbf{F}\left(\mathbf{k J} \cdot \mathrm{mol}^{-1}\right)$
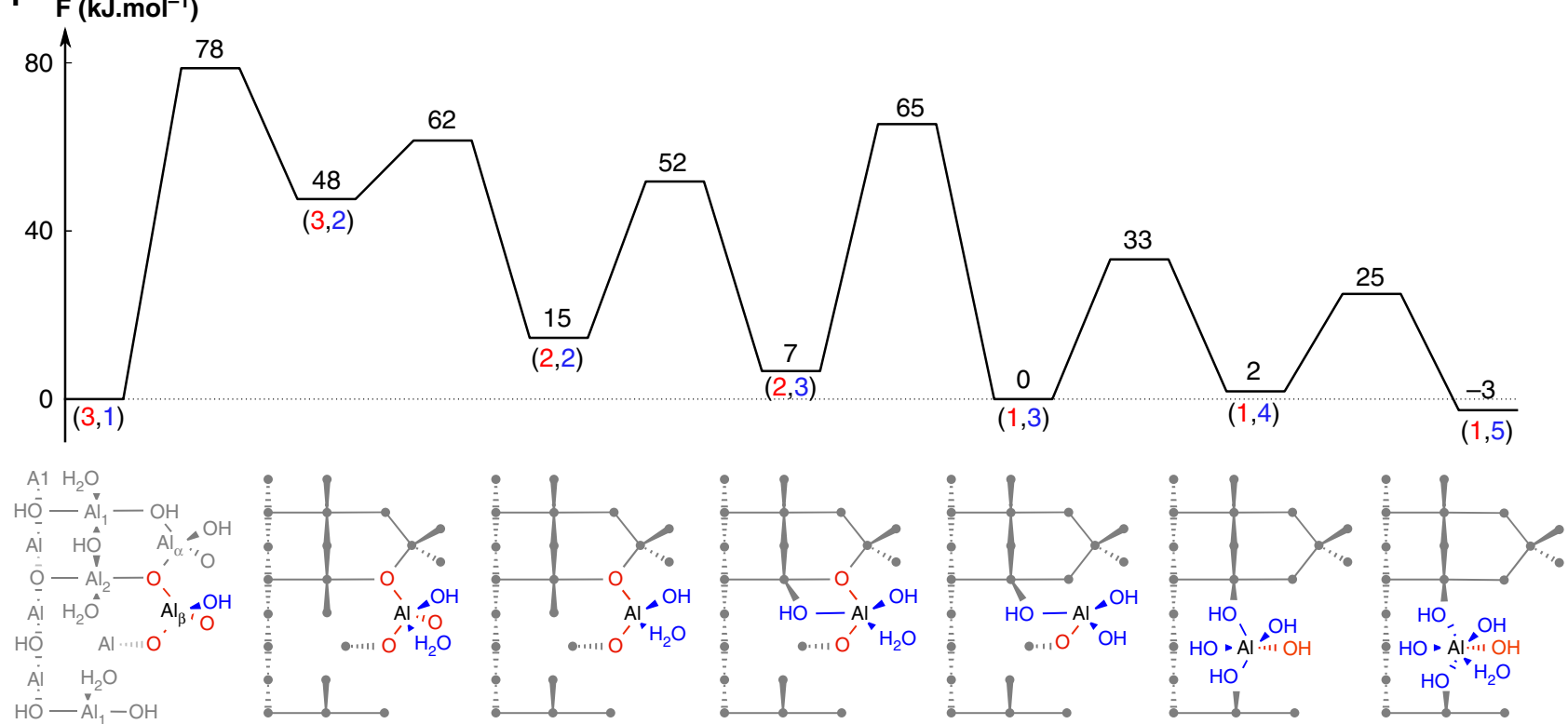

Fig. 2 Exploration of the reactivity of tetrahedral $\mathrm{Al}_{\beta}$ on $\gamma-\mathrm{Al}_{2} \mathrm{O}_{3}(110)$ from ab initio metadynamics. $\mathbf{a}$, $\mathbf{b}$ Structure of the initial surface with chemisorbed water molecules or fragments (the oxygen atoms of which are shown in blue) adsorbed on tetrahedral $\mathrm{Al}_{\alpha}$ and $\mathrm{Al}_{\beta}$ and octahedral $\mathrm{Al}_{(1)}$ and $\mathrm{Al}_{(2)}$. $\mathbf{c} \mathrm{Free}_{\mathrm{C}}$ energy surface obtained from the metadynamics simulation on $\mathrm{Al}_{\beta}$ using the coordination numbers to alumina oxygen atoms $\left(\mathrm{CN}_{\mathrm{a}}\right)$ and to water molecules and fragments $\left(\mathrm{CN}_{\mathrm{w}}\right)$ as variables. The simulation starts from the point of coordinates $\left(\mathrm{CN}_{\mathrm{a}}, \mathrm{CN}_{\mathrm{w}}\right)=(3,1)$. d, e Structure of the last intermediate obtained from the hydration of tetrahedral $\mathrm{Al}_{\beta}$ with $\left(\mathrm{CN}_{\mathrm{a}}, \mathrm{CN}_{\mathrm{w}}\right)=(1,5)$. $\mathbf{f}$ Projected free energy profile with the structure of each intermediate and the corresponding $\left(\mathrm{CN}_{a}, \mathrm{CN}_{\mathrm{w}}\right)$. Yellow and white balls represent aluminum and hydrogen atoms, respectively. The color red is used for alumina oxygen atoms and the associated $\mathrm{CN}_{\mathrm{a}}$. The color blue is used for water oxygen atoms and the associated $\mathrm{CN}_{\mathrm{w}}$

the hydration are accompanied with the deprotonation of some of the coordinated water molecules and a widespread reshuffling of surface protons over about $1 \mathrm{~nm}$ (compare Fig. 2a, d). The amphoteric character of the hydrated material allows $\gamma-\mathrm{Al}_{2} \mathrm{O}_{3}$ to offer the proper local protonation level for the intermediates involved in its own decomposition into $\mathrm{AlO}_{\mathrm{x}} \mathrm{H}_{\mathrm{y}}$. In this respect, the trajectory reveals that physisorbed water molecules are involved, via a Grotthus mechanism, in this redistribution of surface protons. Physisorbed water molecules do therefore not only react but also help accommodating the protonation state of the surface to guest the final octahedral intermediate. This is particularly striking for the water fragments bound to the octahedral $\mathrm{Al}_{(1)}$ and $\mathrm{Al}_{(2)}$, which need to be deprotonated to coordinate the fleeing tetrahedral $\mathrm{Al}_{\beta}$. This study highlights the key role of water as a reactant and as a solvent in the stability of an oxide and pre-figures the role of the $\mathrm{pH}$ in the interface transformation. Ab initio metadynamics can hence provide a complete atomistic picture of the alteration mechanism of an iono-covalent solid in contact with a reactive solvent.

Simulation with chemisorbed xylitol. We have performed the same simulation substituting the water fragments bound to the 
a

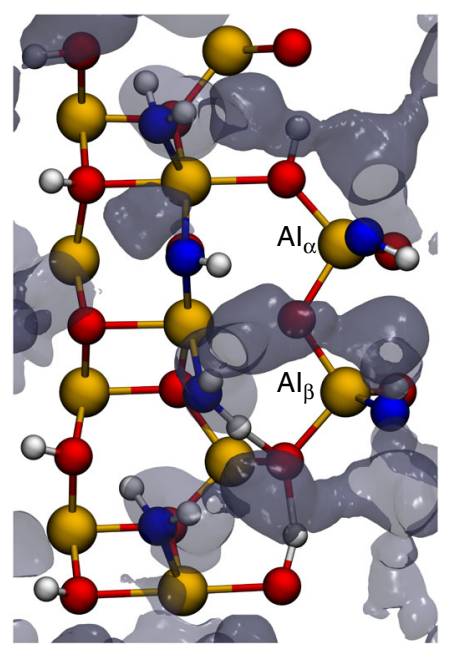

b

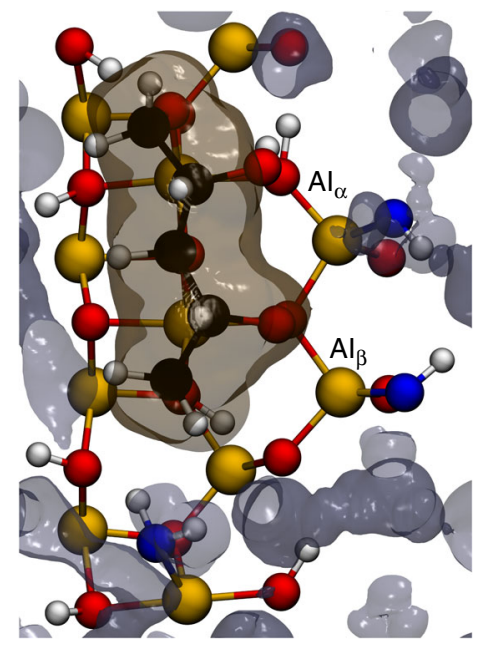

C

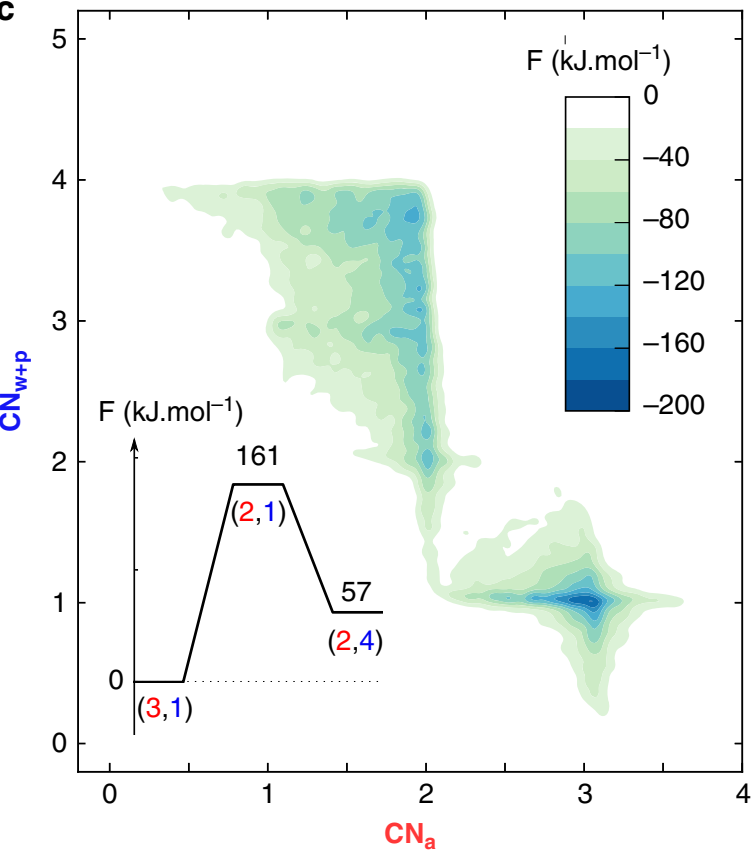

Fig. 3 Inhibition of the decomposition of $\gamma-\mathrm{Al}_{2} \mathrm{O}_{3}$ in the presence of chemisorbed xylitol. a $\gamma-\mathrm{Al}_{2} \mathrm{O}_{3}(110) /$ water interface with the average occupation volume of water in gray. $\mathbf{b}$ Geometry of adsorbed xylitol at the $\gamma-\mathrm{Al}_{2} \mathrm{O}_{3}(110) /$ water interface with the iso-surface of the average occupation volume of xylitol in ochre, and the average occupation volume of water in gray. The iso-surfaces were computed from an extra regular ab initio molecular dynamics simulation. More details can be found in Supplementary Note 8. $\mathbf{c}$ Free energy surface obtained from the metadynamics simulation on $\mathrm{Al}_{\beta}$ in presence of xylitol. $\mathrm{CN}_{a}$ is the coordination number of $A \mathrm{I}_{\beta}$ to alumina oxygen atoms. $\mathrm{CN}_{\mathrm{w}+\mathrm{p}}$ is the coordination number of $\mathrm{Al}_{\beta}$ to water and polyols oxygens. Yellow and white balls represent aluminum and hydrogen atoms, respectively. The color red is used for alumina oxygen atoms and the associated $\mathrm{CN}_{\mathrm{a}}$. The color blue is used for water oxygen atoms and the associated $\mathrm{CN}_{\mathrm{w}}$

octahedral $\mathrm{Al}_{(1)}$ and $\mathrm{Al}_{(2)}$, which are in close vicinity to the watersensitive tetrahedral $\mathrm{Al}_{\beta}$, with xylitol. The choice of the adsorption geometry (Fig. 3b and other views in Supplementary Fig. 7) is globally in agreement with earlier studies on polyol adsorption on alumina (Supplementary Note 8$)^{29}$. The reactivity of $\mathrm{Al}_{\beta}$ is described using again the coordination number of $\mathrm{Al}_{\beta}$ to alumina oxygen atoms $\left(\mathrm{CN}_{\mathrm{a}}\right)$ as a variable. Since now, oxygen atoms are also found in xylitol, we considered, as a second variable, the coordination number to all other oxygen atoms that are external to alumina (oxygen atoms of water molecules and polyol) and referred to as $\mathrm{CN}_{\mathrm{w}+\mathrm{p}}$. The resulting free energy landscape in the presence of xylitol (Fig. 3c) is profoundly modified compared to the uncoated case (Fig. 2c). Despite the exploration of a $200 \mathrm{~kJ}$ $\mathrm{mol}^{-1}$ energy span, the sampled $\left(\mathrm{CN}_{\mathrm{a}}, \mathrm{CN}_{\mathrm{w}+\mathrm{p}}\right)$ space is reduced, meaning that $\mathrm{Al}_{\beta}$ is more constrained near its initial geometry at $\left(\mathrm{CN}_{\mathrm{a}}, \mathrm{CN}_{\mathrm{w}+\mathrm{p}}\right)=(3,1)$. A detailed look at the free energy landscape shows nevertheless that $\mathrm{Al}_{\beta}$ is able to react with some surrounding oxygen atoms $\left(\mathrm{CN}_{\mathrm{w}+\mathrm{p}}\right.$ goes up to 4$)$. The exact mechanism is however very different from that described above. With xylitol adsorbed, $\mathrm{CN}_{\mathrm{a}}$ first decreases from 3 to 2 and $\mathrm{CN}_{\mathrm{w}+\mathrm{p}}$ increases from 1 to 2 , without any clear minimum in-between. This corresponds to a $S_{N} 2$ mechanism with a strong $S_{N} 1$ character where the $\mathrm{Al}_{\beta}$ leaches first from alumina to the outer surface $\left(\mathrm{CN}_{\mathrm{a}}\right.$ decreases) before gaining an $\mathrm{Al}-\mathrm{O}$ bond with water or xylitol $\left(\mathrm{CN}_{\mathrm{w}+\mathrm{p}}\right.$ increases). As discussed above, the hydration of $\mathrm{Al}_{\beta}$ follows an addition/elimination mechanism in the absence of xylitol. In other words, while a water molecule could easily reach the $\mathrm{Al}_{\beta}$ and perform a nucleophilic addition in absence of xylitol, it has become more difficult in presence of xylitol. The nucleophile must therefore either be immobilized or have difficulties to approach $\mathrm{Al}_{\beta}$. This is indeed confirmed by the inspection of the trajectories: $\mathrm{Al}_{\beta}$ does not react with physisorbed water molecules but rather with the chemisorbed alcohol moieties of xylitol. This frustrated extraction of $\mathrm{Al}_{\beta}$ out of the alumina surface is highly activated and shows a barrier of $161 \mathrm{~kJ} \mathrm{~mol}^{-1}$, consistent with the experimentally observed inhibiting power of xylitol for hydroxides formation.

To better understand why $\mathrm{Al}_{\beta}$ reacts with xylitol rather than water, we have performed a 25 ps long regular ab initio molecular dynamics simulation of the interface with xylitol adsorbed and compared to our recently published study work on the $\gamma$ $\mathrm{Al}_{2} \mathrm{O}_{3}(110) /$ water interface ${ }^{33}$. The volume visited by physisorbed water molecules around $\mathrm{Al}_{\beta}$ is greatly impacted by the presence of xylitol as shown in Fig. 3a, b. This is further confirmed by the radial distribution functions of $\mathrm{Al}_{\beta}$ with physisorbed water molecules: the probabilities of finding physisorbed water molecules in the second coordination shell are indeed reduced and shifted to larger distances (Supplementary Fig. 8). This clearly evidences an important steric hindrance induced by xylitol, which is at the origin of the inhibition.

Once $\mathrm{Al}_{\beta}$ sees its coordination number to alumina oxygen atoms diminished by one unit, it directly coordinates to two alcohol groups because of the constrained adsorption mode of xylitol $\left(\mathrm{CN}_{\mathrm{w}+\mathrm{p}}\right.$ increases by two units). The resulting squarebased pyramidal structure readily captures a water physisorbed molecule to gain a saturated octahedral geometry, reaching $\left(\mathrm{CN}_{\mathrm{a}}\right.$, $\left.\mathrm{CN}_{\mathrm{w}+\mathrm{p}}\right)=(2,4)$. Two other adsorption modes of xylitol, which substitute the chemisorbed water molecules involved in the decomposition mechanism, have been tested. They both turn out to be able to inhibit the early stages of the decomposition, proving that the inhibition shown here does not depend on the orientation of the tridentate adsorbate, in spite of mechanistic differences as discussed in Supplementary Note 9.

Noticeably, the inhibitor does not directly interact with the weak spot, but with a neighboring site, thereby modifying the interfacial structure of the liquid. It substitutes the water molecules that are 
involved in the first hydration steps and prevents the necessary proton reshuffling to generate $\mathrm{AlO}_{\mathrm{x}} \mathrm{H}_{\mathrm{y}}$ and therefore provides a strong stabilization of the surface of alumina. This is attributed to the $\gamma$-pentanetriol backbone of xylitol, a structural feature shared with sorbitol (a known good inhibitor) but not with glycerol (a known weak inhibitor) ${ }^{28}$. Our study therefore opens the road to a rational design of the structure of coating agents able to prevent $\gamma$ $\mathrm{Al}_{2} \mathrm{O}_{3}$ from decomposing in water.

In conclusion, the combined experimental and theoretical study presented here provides an atomistic mechanistic picture of the initial steps of the decomposition of $\gamma-\mathrm{Al}_{2} \mathrm{O}_{3}$ in water. The weak spot is a tetrahedral surface $\mathrm{Al}$ of the (110) surface. It undergoes successive addition of water/scission of Al-O, yielding to $\mathrm{AlO}_{\mathrm{x}} \mathrm{H}_{\mathrm{y}}$. This process is accompanied by proton reshuffling. The chemisorption of a polyol on a neighbor site inhibits this hydrolysis by replacing the water molecules that initiate the process and by limiting the access of water to this weak spot. Noticeably, the weak spot of this oxide is not located at kink or edges but at the heart of a given facet. This understanding opens the road to further improvement of inhibitors. Since $\gamma-\mathrm{Al}_{2} \mathrm{O}_{3}$ is an iono-covalent oxide and water potentially reacts during the process and may dissociate into $\left(\mathrm{OH}^{-}, \mathrm{H}^{+}\right)$, gaining such an atomistic understanding is a rather challenging task, especially regarding computational chemistry where the use of biased ab initio molecular dynamic is necessary. The present achievement thus constitutes an unprecedented milestone in the understanding of solid/liquid interface transformation. The approach that we propose here could be insightfully applied to various other relevant systems in electrochemistry, geochemistry or material science, where the reactivity of the solid with water and possibly other liquids plays a crucial role.

\begin{abstract}
Methods
Alumina materials syntheses. Alumina $\mathrm{C}$ (for Commercial) was obtained by calcination of commercial boehmite (Sasol PURAL SB3) at $600^{\circ} \mathrm{C}$ for $4 \mathrm{~h}$. Alumina $\mathrm{F}$ (for Fibers) was synthesized by precipitation of aluminum nitrate ( $\mathrm{Al}$ $\left.\left(\mathrm{NO}_{3}\right)_{3} \cdot 9 \mathrm{H}_{2} \mathrm{O}, 0.1 \mathrm{~mol} \mathrm{~L}^{-1}\right)$ in an aqueous medium. The $\mathrm{pH}$ was adjusted to an initial value of 8 by addition of sodium hydroxide $\left(\mathrm{NaOH}, 1 \mathrm{~mol} \mathrm{~L}^{-1}\right)$ and the resulting suspension was aged at $95^{\circ} \mathrm{C}$ for 1 week. The final $\mathrm{pH}$ value of the suspension was 4.5 . The solid was recovered by centrifugation and washed three times with water. This synthesis was adapted from the method described by Chiche et al. ${ }^{44}$ and Jolivet et al. ${ }^{45}$ Alumina $\mathrm{P}$ (for Plates) and R (for Rods) were obtained by an hydrothermal treatment of alumina $\mathrm{C}$ in water (alumina $\mathrm{P}$ ) or in acidified water with acetic acid (alumina R). Typically, $15 \mathrm{~g}$ of alumina $\mathrm{C}$ were dispersed in $100 \mathrm{~mL}$ of water or acidified water $(\mathrm{pH}=2)$. The mixture was heated at $200{ }^{\circ} \mathrm{C}$ for $10 \mathrm{~h}$ under mechanical stirring and autogeneous pressure in a stainless steel autoclave. During this step, alumina was dissolved and boehmite precipitation occurred. Boehmite nanoparticles properties (size, morphology, texture, etc.) are depending on the experimental conditions ( $\mathrm{pH}$, temperature, concentrations... $)^{45,46}$. In an acidic medium, boehmite precipitates as rod-like nanoparticles. In neutral medium, nanoparticles adopt a plate-like morphology. After cooling at room temperature (RT), the solid phase was recovered by centrifugation, dried at $100^{\circ} \mathrm{C}$ overnight and calcinated at $600{ }^{\circ} \mathrm{C}$ for $4 \mathrm{~h}$.
\end{abstract}

Material characterization. XRD analyses were performed on powders with a Bragg'Brentano diffractometer (PANalytical X'Pert PRO MDP) using $\mathrm{Cu} \mathrm{Ka}$ radiation. Diffractograms were obtained from $2 \theta=4-74^{\circ}$ with a step of $0.033^{\circ}$ and $5 \mathrm{~s}$ per step. Textural properties of aluminas were determined by $\mathrm{N}_{2}$ sorption studies at $77 \mathrm{~K}$ using a Micromeritics ASAP 2000 instrument. The BET method was applied to determine the specific surface area. Transmission electron microscopy (TEM) images were obtained on a JEOL $2010 \mathrm{LaB}_{6}$ microscope operating at $200 \mathrm{kV}$. A dispersion of the sample crushed in ethanol was deposited on standard holey carbon-covered copper TEM grids.

\footnotetext{
Adsorption experiments. Polyol adsorption isotherms were performed on three alumina materials exhibiting different morphologies in a $100 \mathrm{~mL}$ stainless steel autoclave equipped with a mechanical stirring rod (Top Industrie). Aqueous solutions of xylitol and sorbitol $\left(0.5 \mathrm{~g} \mathrm{~L}^{-1}, 1 \mathrm{gL}^{-1}, 2 \mathrm{~g} \mathrm{~L}^{-1}, 4 \mathrm{~g} \mathrm{~L}^{-1}, 6 \mathrm{~g} \mathrm{~L}^{-1}\right.$, and $8 \mathrm{~g}$ $\mathrm{L}^{-1}$ ) prepared using commercial polyols (Sigma Aldrich) and deionized water. Typically, $2 \mathrm{~g}$ of alumina were dispersed in $50 \mathrm{~mL}$ of an aqueous solution of polyol. After $2 \mathrm{~h}$ at $200{ }^{\circ} \mathrm{C}$ under autogeneous pressure (14 bar) and vigorous stirring, a sample of the liquid phase was taken and the solid phase was recovered by
}

centrifugation. Final concentration was determined by high-performance liquid chromatography (HPLC) analysis using a Shimadzu Rezex RXM-Monosaccharide $\mathrm{Ca}^{2+} 8 \%$ column connected to a differential refraction detector (Shimadzu RID10A). The amount of polyol adsorbed was calculated from the difference between initial and final concentrations.

Atomistic model. The model of $\gamma-\mathrm{Al}_{2} \mathrm{O}_{3}(110)$ /water interface was taken from our previous study on the characterization of interfacial water in contact with $\gamma$ $\mathrm{Al}_{2} \mathrm{O}_{3} .{ }^{33}$ This model was built using the surface model proposed by Digne et al. ${ }^{47}$ (and further improved by Wischert et al. ${ }^{48}$ ) and has been used for about 15 years to rationalize efficiently experimental data. ${ }^{19}$ The simulation cell consists of a $10 \AA$ thick $2 \times 2$ unit cell of $\gamma-\mathrm{Al}_{2} \mathrm{O}_{3}$ (110), which is surmounted with a $20 \AA$ thick water layer and another $10 \AA$ thick layer of void to avoid spurious confinement effects ${ }^{49}$

Molecular simulations. All ab initio molecular dynamics simulations (AIMD) reported here were performed using density functional theory (DFT) with the Gaussian and Plane Wave combined approach as implemented in CP2K/Quickstep $^{50-53}$ using the same model and set of parameters as in our previous work on the $\gamma-\mathrm{Al}_{2} \mathrm{O}_{3}(110) /$ water interface ${ }^{33}$. The electrons were treated using the exchange correlation PBE functional ${ }^{54}$ with a Grimme D $3^{55}$ correction. Core electrons were described using the Goedecker-Teter-Hutter (GTH) pseudo-potentials ${ }^{56-58}$ and the valence density was developed on a double-zeta DZVP basis set along with an auxiliary plane wave basis set with cutoff energy of $400 \mathrm{Ry}$.

During AIMD, nuclei were treated within the Born-Oppenheimer approximation with a time step of $0.5 \mathrm{fs}$ for the integration of the equations of motion. The temperature of the simulation was maintained at $330 \mathrm{~K}$ using the Canonical Sampling through Velocity Rescaling (CSVR) thermostat coupled to the system with a time constant of $100 \mathrm{fs}^{59}$. All the systems presented here were thermalized for at least 5 ps before production of about 30 ps for statistical analysis.

For the well-tempered ab initio metadynamics simulations ${ }^{60}$, Gaussian hills of 0.04 width and $3.3 \mathrm{~kJ} \mathrm{~mol}^{-1}$ initial height were added in a two-dimensional set of collective variables, described by coordination numbers $(\mathrm{CN})$ as defined in Plumed $2.0^{61}$. For each collective variable, the chosen $\mathrm{CN}$ gives the average number on a set of oxygen atoms coordinated to the probed $\mathrm{Al}$ atom. We used two different sets. In the first one $\left(\mathrm{CN}_{\mathrm{a}}\right)$, the oxygen atoms belong to the surface structure of dry alumina. The second one includes all the other oxygen atoms of the simulations $\left(\mathrm{CN}_{\mathrm{w}}\right.$ or $\mathrm{CN}_{\mathrm{w}+\mathrm{p}}$, with $\mathrm{w}$ for water oxygens and $\mathrm{p}$ for polyol oxygens, respectively) The error associated with this procedure is lower than $2 \mathrm{~kJ} \mathrm{~mol}^{-1}$. Further details are given in Supplementary Notes 6-9.

\section{Data Availability}

The source data underlying Fig. $1 \mathrm{~d}-\mathrm{f}$ are available in SI. Raw data were generated at the PSMN and GENCI large-scale facilities. Derived data supporting the findings of this study are available as supplementary materials: a snapshot of each ab initio MD is provided as well as the grid-based data of the Free Energy Surface that are plotted in Figs. $2 c$ and $3 c$.

Received: 8 January 2019 Accepted: 17 May 2019

Published online: 17 July 2019

\section{References}

1. Sato, H., Miyagawa, Y., Okabe, T., Miyajima, M. \& Sunada, H. Dissolution mechanism of diclofenac sodium from wax matrix granules. J. Pharm. Sci. 86, 929-934 (1997).

2. Maurice, V. \& Marcus, P. Progress in corrosion science at atomic and nanometric scales. Prog. Mater. Sci. 95, 132-171 (2018).

3. Chen, Z., Amine, R., Ma, Z. -F. \& Amine, K. Interfacial reactions in lithium batteries. J. Phys. D. Appl. Phys. 50, 303001 (2017).

4. Fischer, C., Kurganskaya, I., Schäfer, T. \& Lüttge, A. Variability of crystal surface reactivity: what do we know? Appl. Geochemistry 43, 132-157 (2014).

5. Putnis, A. \& Putnis, C. V. The mechanism of reequilibration of solids in the presence of a fluid phase. J. Solid State Chem. 180, 1783-1786 (2007).

6. Pirkanniemi, K. \& Sillanpää, M. Heterogeneous water phase catalysis as an environmental application: a review. Chemosphere 48, 1047-1060 (2002).

7. Chheda, J. N., Huber, G. W. \& Dumesic, J. A. Liquid-phase catalytic processing of biomass-derived oxygenated hydrocarbons to fuels and chemicals. Angew. Chemie Int. Ed. 46, 7164-7183 (2007).

8. Sievers, C. et al. Phenomena affecting catalytic reactions at solid-liquid interfaces. ACS Catal. 6, 8286-8307 (2016).

9. Skorina, T. \& Allanore, A. Aqueous alteration of potassium-bearing aluminosilicate minerals: from mechanism to processing. Green. Chem. 17, 2123-2136 (2015).

10. Chen, G. et al. Successive interfacial reaction-directed synthesis of $\mathrm{CeO} 2$ @Au@CeO 2 -MnO 2 environmental catalyst with sandwich hollow structure. ACS Appl. Mater. Interfaces 10, 11595-11603 (2018). 
11. Zhang, X., Sewell, T. E., Glatz, B., Sarupria, S. \& Getman, R. B. On the water structure at hydrophobic interfaces and the roles of water on transition-metal catalyzed reactions: a short review. Catal. Today 285, 57-64 (2017).

12. Ravenelle, R. M., Copeland, J. R., Kim, W. -G., Crittenden, J. C. \& Sievers, C. Structural changes of $\gamma$-Al 2 O 3 -supported catalysts in hot liquid water. ACS Catal. 1, 552-561 (2011).

13. Hilfiker, R. Polymorphism: In the Pharmaceutical Industry (Wiley-VCH, Weinheim, Germany, 2006).

14. Siegfried, M. J. \& Choi, K. -S. Elucidating the effect of additives on the growth and stability of $\mathrm{Cu} 2 \mathrm{O}$ surfaces via shape transformation of pre-grown crystals. J. Am. Chem. Soc. 128, 10356-10357 (2006).

15. Pedreira-Segade, U., Michot, L. J. \& Daniel, I. Effects of salinity on the adsorption of nucleotides onto phyllosilicates. Phys. Chem. Chem. Phys. 20, 1938-1952 (2018).

16. Abi Aad, J. et al. Chemical weathering of alumina in aqueous suspension at ambient pressure: a mechanistic study. ChemCatChem 9, 2186-2194 (2017).

17. Busca, G. Structural, surface, and catalytic properties of aluminas. Adv. Catal. 57, 319-404 (2014).

18. Trueba, M. \& Trasatti, S. P. $\gamma$-alumina as a support for catalysts: a review of fundamental aspects. Eur. J. Inorg. Chem. 3393-3403. https://doi.org/10.1002/ ejic.200500348 (2005).

19. Raybaud, P. et al. From $\gamma$-alumina to supported platinum nanoclusters in reforming conditions: 10 years of DFT modeling and beyond. J. Catal. 308, 328-340 (2013).

20. Busca, G. The surface of transitional aluminas: a critical review. Catal. Today 226, 2-13 (2014).

21. Mager-Maury, C., Chizallet, C., Sautet, P. \& Raybaud, P. Platinum nanoclusters stabilized on $\gamma$-alumina by chlorine used as a capping surface ligand: a density functional theory study. ACS Catal. 2, 1346-1357 (2012).

22. Sakwa-Novak, M. A. \& Jones, C. W. Steam induced structural changes of a poly(ethylenimine) impregnated $\gamma$-alumina sorbent for CO 2 extraction from ambient air. ACS Appl. Mater. Interfaces 6, 9245-9255 (2014).

23. Lefèvre, G., Duc, M., Lepeut, P., Caplain, R. \& Fédoroff, M. Hydration of $\gamma$ alumina in water and its effects on surface reactivity. Langmuir 18, 7530-7537 (2002).

24. Carrier, X., Marceau, E., Lambert, J. -F. \& Che, M. Transformations of $\gamma$ alumina in aqueous suspensions 1 . Alumine chemical weathering as a function of pH. J. Colloid Interface Sci. 308, 429-437 (2007).

25. Jongerius, A. L. et al. Stability of $\mathrm{Pt} / \gamma-\mathrm{Al} 2 \mathrm{O} 3$ catalysts in lignin and lignin model compound solutions under liquid phase reforming reaction conditions. ACS Catal. 3, 464-473 (2013).

26. Liu, F. et al. Silica deposition as an approach for improving the hydrothermal stability of an alumina support during glycerol aqueous phase reforming. Appl. Catal. A Gen. 551, 13-22 (2018).

27. Abi Aad, J. et al. Inhibition by inorganic dopants of $\gamma$-Al2O3 chemical weathering in hydrothermal conditions: identification of reactive sites and influence in Fischer-Tropsch synthesis. Chem. Cat. Chem. 9, 2106-2117 (2017).

28. Ravenelle, R. M., Copeland, J. R., Van Pelt, A. H., Crittenden, J. C. \& Sievers, C. Stability of $\mathrm{Pt} / \gamma$-Al2O3 catalysts in model biomass solutions. Top. Catal. 55, 162-174 (2012).

29. Copeland, J. R., Shi, X. -R., Sholl, D. S. \& Sievers, C. Surface interactions of C2 and $\mathrm{C} 3$ polyols with $\gamma$-Al2O3 and the role of coadsorbed water. Langmuir 29, 581-593 (2013).

30. Adiga, S. P., Zapol, P. \& Curtiss, L. A. Atomistic simulations of amorphous alumina surfaces. Phys. Rev. B 74, 064204 (2006).

31. Krokidis, X. et al. Theoretical study of the dehydration process of boehmite to $\gamma$-alumina. J. Phys. Chem. B 105, 5121-5130 (2001).

32. Ngouana-Wakou, B. F., Cornette, P., Corral Valero, M., Costa, D. \& Raybaud, P. An atomistic description of the $\gamma$-alumina/water interface revealed by $a b$ initio molecular dynamics. J. Phys. Chem. C. 121, 10351-10363 (2017).

33. Réocreux, R., Jiang, T., Iannuzzi, M., Michel, C. \& Sautet, P. Structuration and dynamics of interfacial liquid water at hydrated $\gamma$-alumina determined by $a b$ initio molecular simulations: implications for nanoparticle stability. ACS Appl. Nano Mater. 1, 191-199 (2018).

34. Laio, A. \& Gervasio, F. L. Metadynamics: a method to simulate rare events and reconstruct the free energy in biophysics, chemistry and material science. Rep. Prog. Phys. 71, 126601 (2008).

35. Michel, C. et al. Free energy ab initio metadynamics: a new tool for the theoretical study of organometallic reactivity? example of the $\mathrm{C}-\mathrm{C}$ and $\mathrm{C}-\mathrm{H}$ reductive eliminations from platinum(IV) complexes. Organometallics 26, 1241-1249 (2007).

36. Blumberger, J., Ensing, B. \& Klein, M. L. Formamide hydrolysis in alkaline aqueous solution: insight from ab initio metadynamics calculations. Angew. Chem. -Int. Ed. 45, 2893-2897 (2006).

37. Moors, S. L. C., De Wispelaere, K., Van der Mynsbrugge, J., Waroquier, M. \& Van Speybroeck, V. Molecular dynamics kinetic study on the zeolite-catalyzed benzene methylation in ZSM-5. ACS Catal. 3, 2556-2567 (2013).
38. Muñoz-Santiburcio, D., Farnesi Camellone, M. \& Marx, D. Solvation-Induced changes in the mechanism of alcohol oxidation at gold/titania nanocatalysts in the aqueous phase versus gas phase. Angew. Chemie Int. Ed. 57, 3327-3331 (2018).

39. Stack, A. G., Raiteri, P. \& Gale, J. D. Accurate rates of the complex mechanisms for growth and dissolution of minerals using a combination of rare-event theories. J. Am. Chem. Soc. 134, 11-14 (2012).

40. Salvalaglio, M., Vetter, T., Giberti, F., Mazzotti, M. \& Parrinello, M. Uncovering molecular details of urea crystal growth in the presence of additives. J. Am. Chem. Soc. 134, 17221-17233 (2012).

41. Sprycha, R. Electrical double layer at alumina/electrolyte interface. J. Colloid. Interface Sci. 127, 1-11 (1989).

42. Kosmulski, $\mathrm{M}$. The $\mathrm{pH}$-dependent surface charging and points of zero charge. J. Colloid. Interface Sci. 353, 1-15 (2011).

43. Silaghi, M. -C., Chizallet, C., Sauer, J. \& Raybaud, P. Dealumination mechanisms of zeolites and extra-framework aluminum confinement. J. Catal. 339, 242-255 (2016).

44. Chiche, D., Digne, M., Revel, R., Chanéac, C. \& Jolivet, J. -P. Accurate determination of oxide nanoparticle size and shape based on X-Ray powder pattern simulation: application to boehmite AlOOH. J. Phys. Chem. C. 112, 8524-8533 (2008)

45. Jolivet, J. -P. et al. Size tailoring of oxide nanoparticles by precipitation in aqueous medium. A semi-quantitative modelling. J. Mater. Chem. 14, 3281-3288 (2004).

46. He, T., Xiang, L. \& Zhu, S. Different nanostructures of boehmite fabricated by hydrothermal process: effects of $\mathrm{pH}$ and anions. CrystEngComm 11, 1338 (2009).

47. Digne, M., Sautet, P., Raybaud, P., Euzen, P. \& Toulhoat, H. Hydroxyl groups on $\gamma$-alumina surfaces: a DFT study. J. Catal. 211, 1-5 (2002).

48. Wischert, R., Laurent, P., Copéret, C., Delbecq, F. \& Sautet, P. $\gamma$-alumina: the essential and unexpected role of water for the structure, stability, and reactivity of "defect" sites. J. Am. Chem. Soc. 134, 14430-14449 (2012).

49. Bellarosa, L., García-Muelas, R., Revilla-López, G. \& López, N. Diversity at the water-metal interface: metal, water thickness, and confinement effects. ACS Cent. Sci. 2, 109-116 (2016)

50. VandeVondele, J. et al. Quickstep: fast and accurate density functional calculations using a mixed Gaussian and plane waves approach. Comput. Phys. Commun. 167, 103-128 (2005).

51. VandeVondele, J. \& Hutter, J. Gaussian basis sets for accurate calculations on molecular systems in gas and condensed phases. J. Chem. Phys. 127, 114105 (2007)

52. Lippert, G., Hutter, J. \& Parrinello, M. A hybrid Gaussian and plane wave density functional scheme. Mol. Phys. 92, 477-487 (1997).

53. Hutter, J., Iannuzzi, M., Schiffmann, F. \& VandeVondele, J. cp2k: atomistic simulations of condensed matter systems. Wiley Interdiscip. Rev. Comput. Mol. Sci. 4, 15-25 (2014).

54. Perdew, J. P., Burke, K. \& Ernzerhof, M. Generalized gradient approximation made simple. Phys. Rev. Lett. 77, 3865-3868 (1996).

55. Grimme, S., Antony, J., Ehrlich, S. \& Krieg, H. A consistent and accurate ab initio parametrization of density functional dispersion correction (DFT-D) for the 94 elements H-Pu. J. Chem. Phys. 132, 154104 (2010).

56. Goedecker, S., Teter, M. \& Hutter, J. Separable dual-space Gaussian pseudopotentails. Phys. Rev. B 54, 1703-1710 (1996).

57. Hartwigsen, C., Goedecker, S. \& Hutter, J. Relativistic separable dual-space Gaussian pseudopotentials from H to Rn. Phys. Rev. B 58, 3641-3662 (1998)

58. Krack, M. Pseudopotentials for $\mathrm{H}$ to $\mathrm{Kr}$ optimized for gradient-corrected exchange-correlation functionals. Theor. Chem. Acc. 114, 145-152 (2005).

59. Bussi, G., Donadio, D. \& Parrinello, M. Canonical sampling through velocity rescaling. J. Chem. Phys. 126, 014101 (2007).

60. Barducci, A., Bussi, G. \& Parrinello, M. Well-tempered metadynamics: a smoothly converging and tunable free-energy method. Phys. Rev. Lett. 100, 020603 (2008).

61. Tribello, G. A., Bonomi, M., Branduardi, D., Camilloni, C. \& Bussi, G. PLUMED 2: New feathers for an old bird. Comput. Phys. Commun. 185, 604-613 (2014).

\section{Acknowledgements}

We are grateful to the Center Blaise Pascal and the Pôle Scientifique de Modélisation Numérique at the École Normale Supérieure de Lyon for HPC resources. We also thank also the SYSPROD project and AXELERA Pôle de Compétitivité for financial support (PSMN Data Center). This work was granted access to the HPC resources of IDRIS under the allocation 2017-A0010800609 made by GENCI.

\section{Author contributions}

S.P. and M.C. conceived and supervised the project. R.R. conducted the ab initio molecular dynamic simulations. C.P. computed the average occupation volume. G.E. conducted the experimental work that was supervised by T.A., B.M., C.A. and C.A. All the authors discussed the results and commented on the manuscript. R.R. and M.C. prepared the manuscript with inputs from C.P., G.E., T.A., B.M., and S.P. 


\section{Additional information}

Supplementary Information accompanies this paper at https://doi.org/10.1038/s41467019-10981-9.

Competing interests: The authors declare no competing interest.

Reprints and permission information is available online at http://npg.nature.com/ reprintsandpermissions/

Peer review information: Nature Communications thanks the anonymous reviewers for their contribution to the peer review of this work. Peer reviewer reports are available.

Publisher's note: Springer Nature remains neutral with regard to jurisdictional claims in published maps and institutional affiliations. (c) (i) Open Access This article is licensed under a Creative Commons Attribution 4.0 International License, which permits use, sharing, adaptation, distribution and reproduction in any medium or format, as long as you give appropriate credit to the original author(s) and the source, provide a link to the Creative Commons license, and indicate if changes were made. The images or other third party material in this article are included in the article's Creative Commons license, unless indicated otherwise in a credit line to the material. If material is not included in the article's Creative Commons license and your intended use is not permitted by statutory regulation or exceeds the permitted use, you will need to obtain permission directly from the copyright holder. To view a copy of this license, visit http://creativecommons.org/ licenses/by/4.0/.

(C) The Author(s) 2019 